\title{
Optical pulse compression based on enhanced frequency chirping
}

\author{
D. Grischkowsky and A. C. Balant \\ IBM Thomas J. Watson Research Center, P. O. Box 218, Yorktown Heights, New York 10598
}

\begin{abstract}
(Received 24 February 1982; accepted for publication 7 April 1982)
Through numerical simulations, we show that, under relatively general conditions, passage of an intense picosecond pulse through a single-mode optical fiber can cause the pulse to become strongly frequency broadened with a positive chirp (linear frequency sweep) describing essentially all of the energy of the output pulse. Also, because the optical fiber supports only a single transverse mode, the entire output beam profile has the same frequency modulation. These two features allow for unprecedented optical pulse compression.
\end{abstract}

PACS numbers: $42.65 .-\mathrm{k}, 42.80 . \mathrm{Mv}$

Most optical pulse compression schemes are based on the idea originally developed for chirp radar whereby a frequency swept pulse is sent through a linearly dispersive delay line. ${ }^{1-8}$ Since the group velocity of the light is determined by its instantaneous frequency, different portions of the pulse travel at different speeds through the delay line. If the length of the line is adjusted so that the leading edge of the pulse is delayed by just the right amount to overlap the trailing edge at the output of the delay line, the output pulse can be as short as the reciprocal of the bandwidth of the frequency sweep.

The use of this scheme requires a method for producing a linearly frequency swept (chirped) optical pulse. For picosecond optical pulses, it is quite difficult, using electro-optic techniques, to obtain the required magnitude of frequency sweep. Consequently, applications to date have used either the chirp produced by the mode-locked laser itself, ${ }^{2}$ or the chirp produced by the nonlinear process of self-phase modulation ${ }^{3,4,7}$ (SPM) in a nonlinear optical material. For the simplest SPM case, the instantaneous frequency is proportional to the time derivative of the optical pulse shape. Thus, as illustrated in Fig. 1, only the central region of the pulse has the proper chirp for subsequent pulse compression, whereas the opposite chirp on the wings of the pulse will lead to pulse expansion (temporal broadening). Another problem with SPM is that, because of the laser beam's varying spatial intensity profile, the magnitude of the chirp will vary with distance from the center of the beam. This prevents optimal compression of the entire beam profile at any given dispersive delay setting.

In this letter we present a new approach to chirping picosecond pulses, namely by propagating them through a single-mode optical fiber. During passage through the fiber, both the pulse shape and the frequency bandwidth are broadened by the combined action of self-phase modulation (with positive $n_{2}$ ) and positive group velocity dispersion. Under easily achieved conditions of pulse intensity and fiber length, essentially the entire output pulse can be positively, linearly chirped (enhanced frequency chirping). Moreover, because of the single-mode propagation in the fiber, ${ }^{9-11}$ the entire output beam has the same chirp, i.e., the chirp is independent of transverse position on the output beam. This approach made possible the measurement technique introduced in a recent nonlinear pulse propagation experiment ${ }^{11}$ involving optical fibers. Our purpose here is to illustrate by both a simple discussion and a detailed calculation the usefulness and importance of this method to pulse compression applications. We will show that the enhanced frequency chirp enables the output pulse to be subsequently compressed to the frequency transform limit with greatly reduced wings on the compressed pulse, while the lack of any spatial effects in the frequency modulation allows the entire pulse to compress as a spatial unit.

To estimate the operating conditions required to obtain the enhanced chirping of the entire output pulse from the
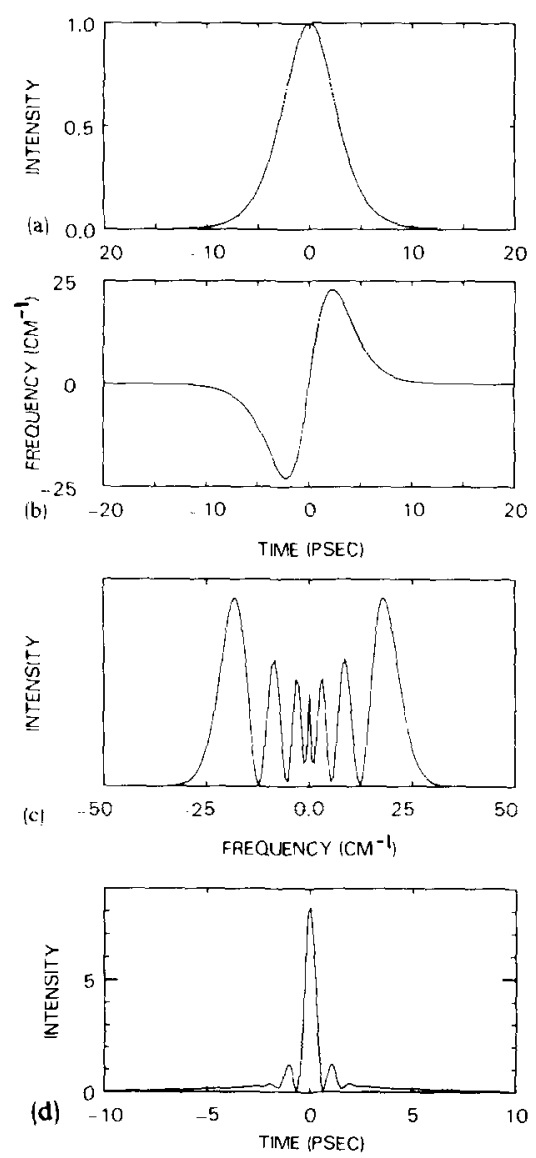

FIG. 1. (a) (Hyperbolic secant) ${ }^{2}$ pulse shape with 6-ps pulsewidth. (b) Selfphase modulation proportional to the derivative of the pulse shape. Frequency in $\mathrm{cm}^{-1}\left(1 \mathrm{~cm}^{-1}=30 \mathrm{GHz}\right)$. (c) Spectral intensity of the Fourier transform of the pulse. (d) Calculated compressed pulse. 
fiber, we present the following simple discussion. Firstly, to be able to obtain significant pulse compression, the bandwidth of the pulse must be substantially increased. Secondly, in order for the output pulse to be described by a single linear chirp, the pulse shape must be markedly changed by the combined action of self-phase modulation and positive group velocity dispersion (GVD). We call this combined process "dispersive self-phase modulation" (DSPM). Enhanced frequency chirping will be possible if DSPM is strong enough to produce significant frequency broadening before the power is reduced to the linear range by the well-known pulsewidth broadening action of GVD. In order to clarify these conditions, we introduce here (and will calculate below) two propagation lengths $Z_{L}$ and $Z_{s}$, where $Z_{L}$ is the length required for the input pulsewidth to double due only to GVD and $Z_{s}$ is the length required for the pulse edges to become arbitrarily sharp due to DSPM. For very short propagation lengths $\left(Z \ll Z_{s} \ll Z_{L}\right)$, significant changes can occur in the pulse spectrum due to SPM with no changes in the pulse shape. If the path length is increased, so that $Z \sim Z_{s} \ll Z_{L}$, DSPM can produce square, self-steepened output pulses. In this case the frequency chirped portion of the pulse contains most of the pulse energy, and optimum compression can be achieved. Much longer propagation paths $\left(Z>Z_{s}\right)$ merely broaden the pulse without significantly increasing the chirp.

From the work of Marcuse ${ }^{12}$ and Fisher and Bischel ${ }^{6}$ we obtain $Z_{L}$ for unmodulated pulses as

$$
Z_{L}=\sqrt{3} \Delta t_{0} /\left(k_{2} \Delta \omega_{0}\right)
$$

$\Delta t_{0}$ is the full width at half-maximum (FWHM) pulsewidth, $\Delta \omega_{0}$ is the FWHM bandwidth, $k_{2}=\partial^{2} k / \partial \omega^{2}=\partial\left(1 / v_{g}\right) / \partial \omega$ is the fiber GVD. To calculate $Z_{s}$ we use a simple picture introduced by Fisher and Bischel, ${ }^{6}$ where SPM and GVD are considered separately. The pulse is first considered to pass undistorted through a region of strong nonlinearity where only SPM occurs. The resulting instantaneous frequency $\omega^{\prime}$ is given by ${ }^{13}$

$$
\omega^{\prime}=\omega_{0}-\frac{\omega_{0}}{c} Z \frac{n_{2}}{2} \frac{\partial E^{2}}{\partial t} .
$$

Here, $\omega_{0}$ is the carrier frequency, $n_{2}$ is the nonlinear index of refraction, and $E$ is the electric field amplitude. Then, the SPM pulse propagates through a strictly linear material with positive GVD, where the edges of the pulse sharpen and reach a singularity at $Z_{s}, 5,8$

$$
1 / Z_{s}=-k_{2} \frac{\partial \omega^{\prime}}{\partial t}
$$

where the time derivative is evaluated at the input to the linear section. ${ }^{14}$

We will now consider the example of a 100-W, 6-ps (FWHM), transform-limited, (hyperbolic secant) $)^{2}$ input pulse to a single-mode optical fiber with a $4-\mu \mathrm{m}$ core diameter, a GVD of $2.5 \mathrm{ps} / \AA$ for a $70-\mathrm{m}$ length, ${ }^{11}$ and an $n_{2}$ of $1.1 \times 10^{-13}$ esu. ${ }^{10,11,13}$ From Eq. (1), $Z_{L}$ is evaluated to be $Z_{L}=480 \mathrm{~m}$. Enhanced frequency chirping with substantial bandwidth broadening will be possible if $Z_{s}$ is much less than $Z_{L}$. In order to estimate the SPM, we use Eq. (2) with $Z=Z_{s} / 2$. Then, using this SPM pulse as the input to the linear section of the fiber, we calculate $Z_{s}$ from Eq. (3) to be $Z_{s}=14 \mathrm{~m}$. Clearly, this is an acceptable value. We would expect from our experience with both experiments and numerical calculations that the optimal chirp would be produced at about $2 Z_{s}$.

As a more precise description of nonlinear pulse propagation in optical fibers we performed a numerical integration of the reduced wave equations for the input pulse of the above example. The equations describing the propagation of the electric field amplitude $E$ and the phase $\phi$ through the single-mode fiber are ${ }^{9-11}$

$$
\begin{aligned}
& \frac{\partial E}{\partial Z}+k_{1} \frac{\partial E}{\partial t}=-\frac{k_{2}}{2}\left(2 \frac{\partial \phi}{\partial t} \frac{\partial E}{\partial t}+E \frac{\partial^{2} \phi}{\partial t^{2}}\right) \\
& \frac{\partial \phi}{\partial Z}+k_{1} \frac{\partial \phi}{\partial t}=+\frac{k_{2}}{2}\left(\frac{1}{E} \frac{\partial^{2} E}{\partial t^{2}}-\left(\frac{\partial \phi}{\partial t}\right)^{2}\right)-\kappa E^{2},(4 b)
\end{aligned}
$$

where the propagating electric field is given by

$$
\mathbf{E}=E \cos \left(k_{0} z-\omega_{0} t-\phi\right) .
$$

In Eqs. (4) and (5), $k_{1}=\partial k / \partial \omega=1 / v_{g}, \kappa=0.5 k_{0} n_{2} / n_{0}$, $k_{0}=2 \pi n_{0} / \lambda_{0}$ is the propagation vector for the carrier wave with the index of refraction $n_{0}$ and the vacuum wavelength $\lambda_{0}=5870 \AA$. The fact that the plane-wave Eqs. (4) have been experimentally shown to describe nonlinear propagation in a single-mode fiber ${ }^{9-11}$ demonstrates that SPM is independent of transverse position on the profile of the propagating beam.
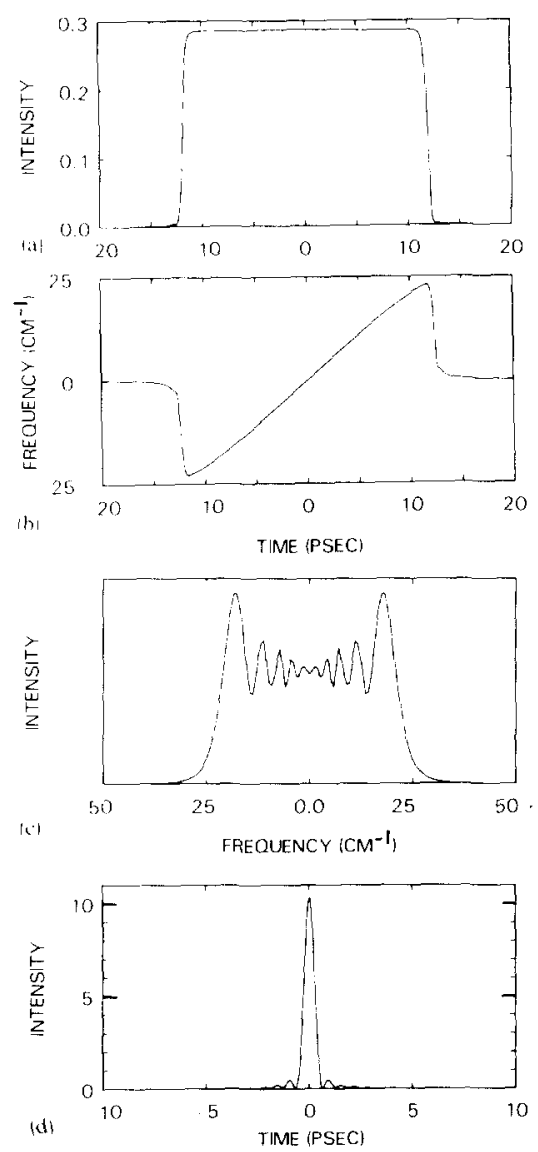

FIG. 2. (a) Calculated DSPM output pulse from $30 \mathrm{~m}$ of optical fiber. (b) Frequency modulation of the output pulse. (c) Spectral intensity of the Fourier transform of the output pulse. (d) Calculated compressed pulse. 
As the pulse propagates through the fiber the calculated reshaping proceeds smoothly from the transform-limited input pulse shown in Fig. 1(a) to the characteristic square pulse shape shown in Fig. 2(a), which occurs at $30 \mathrm{~m}$. Simultaneously, the developing chirp grows in magnitude and describes more and more of the total pulse extent. For this example, the chirp shown in Fig. 2(b) describes more than $95 \%$ of the total energy, compared to only $59 \%$ for the selfphase modulated pulse shown in Fig. 1. In agreement with our simple discussion, enhanced frequency chirping occurs for the propagation distance $Z \sim 2 Z_{s}$, where for this example $Z=30 \mathrm{~m}$ and $Z_{s}=14 \mathrm{~m}$. Our numerical calculations also show that enhanced chirping is obtained for fiber lengths greater than $Z_{s}$. This demonstrates the practical feasibility of the method. However, for distances longer than $2 Z_{s}$ the pulse continues to broaden but the magnitude of the chirp does not increase significantly and little is gained by using longer fibers. Thus, $Z \sim 2 Z_{s}$ is a reasonable estimate for the optimal fiber length.

These relationships were initially demonstrated experimentally in Ref. 11, where enhanced chirping was obtained for 10-W, 5.5-ps, (hyperbolic secant) ${ }^{2}$ pulses propagated through a 70-m fiber for which $Z_{s}$ is calculated to be $40 \mathrm{~m}$. These pulses were subsequently compressed to 1.5 ps by passage through a sodium vapor cell. More recently, Shank $e t$ $a l .{ }^{15}$ used this technique to produce the shortest optical pulses ( $30 \mathrm{fs}$ ) to date. For their experiment 7-kW, 90-fs (hyperbolic secant) $)^{2}$ pulses were propagated through a $15-\mathrm{cm}$ fiber for which $Z_{s}$ is calculated to be $2.3 \mathrm{~cm}$. These pulses were subsequently compressed to $30 \mathrm{fs}$ by passage through a grating pair. In agreement with their calculations, good compression would also have been obtained with $Z \sim 2 Z_{s}$.

The pulse compression obtainable with the fiber output pulse (DSPM pulse) of Fig. 2(a) will now be compared to that for the pure SPM case of Fig. 1. For this comparison the (hyperbolic secant) ${ }^{2}$ input pulses and the magnitude of the frequency modulation [Figs. $1(\mathrm{~b})$ and $2(\mathrm{~b})]$ are the same. These pulses are Fourier analyzed [Figs. 1(c) and 2(c)] and considered to pass through dispersive delay lines with group velocity dispersions opposite to that of the optical fiber. At the output of the delay lines the Fourier components are summed to give the compressed pulses. The lengths of the delay lines are adjusted to obtain the shortest pulses shown in Figs. 1(d) and 2(d). For both cases the compressed pulsewidths are approximately 0.6 ps compared with the in put pulsewidths of $6 \mathrm{ps}$, and the intensities of the compressed pulses have correspondingly increased by about 10 times. The intensity of the compressed DSPM pulse is higher than the SPM pulse because the central peak of the compressed DSPM pulse contains more of the total energy. Furthermore, the compressed DSPM pulse has much less extensive wings than the SPM pulse, as illustrated by the expanded scale of Fig. 3. The relative energies in the wings of the two compressed pulses, as measured from the null closest to the central peaks, are $42 \%$ for the SPM pulse and only $10.6 \%$

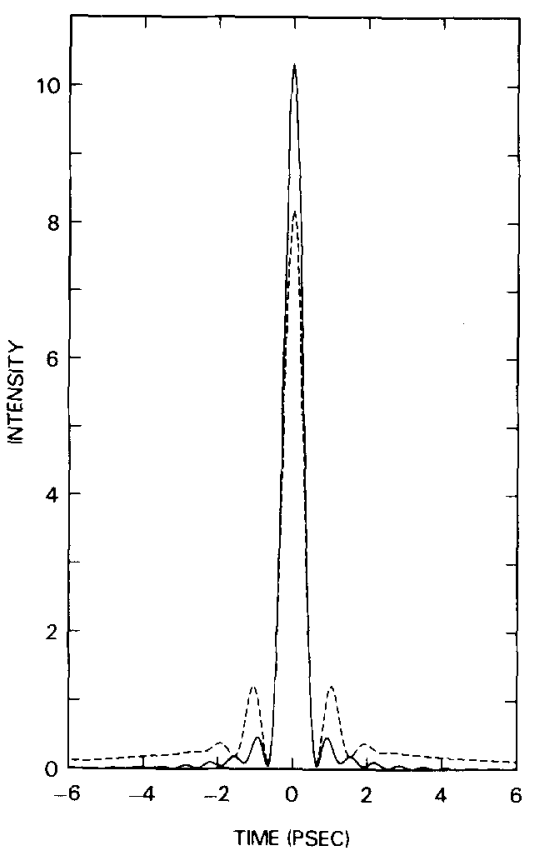

FIG. 3. Comparison of the compressed pulses of Figs. 1(d) and 2(d). Solid line is the DSPM pulse; dashed line is the SPM pulse.

for the DSPM pulse. The ratio of the intensity of the main pulse to the secondary maxima (pulse lobes) is 22 for the DSPM pulse and 7 for the SPM pulse. For the extended wings measured from \pm 2 ps the relative energies are $25 \%$ for the SPM pulse compared to only $3 \%$ for the DSPM pulse. The significantly reduced wings resulting from enhanced frequency chirping should prove important to the applications of these compressed DSPM pulses.

We would like to acknowledge helpful comments by J. J. Wynne concerning this manuscript. This work was partially supported by the U. S. Office of Naval Research.

'J. R. Klauder, A. C. Price, S. Darlington, and W. J. Albersheim, Bell Syst. Tech. J. 39, $745(1960)$.

${ }^{2}$ E. B. Treacy, Phys. Lett. 28A, $34(1968)$.

${ }^{3}$ R. A. Fisher, P. L. Kelley, and T. K. Gustafson, Appl. Phys. Lett. 14, 140 (1969).

${ }^{4}$ A. Laubereau, Phys. Lett. 29A, 539 (1969).

${ }^{5}$ D. Grischkowsky, Appl. Phys. Lett. 25, 566 (1974).

${ }^{6}$ R. A. Fisher and W. K. Bischel, J. Appl. Phys. 46, 4921 (1975).

${ }^{7}$ R. H. Lehmberg and J. M. McMahon, Appl. Phys. Lett. 28, 204 (1976); R.

H. Lehmberg, J. Reintjes, and R. C. Eckardt, Opt. Commun. 22, 95 (1977).

${ }^{\circledR} \mathrm{J}$. K. Wigmore and D. Grischkowsky, IEEE J. Quantum Electron. QE-14, $310(1978)$. This paper contains an extensive listing of the pulse compression literature.

${ }^{9}$ A. Hasegawa and F. Tappert, Appl. Phys. Lett. 23, 142 (1973).

${ }^{10}$ L. F. Mollenauer, R. H. Stolen, and J. P. Gordon, Phys. Rev. Lett. 45, 1095 (1980).

"H. Nakatsuka, D. Grischkowsky, and A. C. Balant, Phys. Rev. Lett. 47, 910 (1981).

${ }^{12}$ D. Marcuse, Appl. Opt. 19, 1653 (1980).

${ }^{13}$ R. H. Stolen and C. Lin, Phys. Rev. A 17, 1448 (1978).

${ }^{14}$ For a Gaussian pulse our $Z_{s} \sim Z_{\text {shock }} / 3$ of Ref. 6 .

${ }^{15}$ C. V. Shank, R. L. Fork, R. Yen, R. H. Stolen, and W. J. Tomlinson, Appl. Phys. Lett. 40, 761 (1982). 